\title{
Guidelines: l'évidence comme pierre angulaire *
}

Consensus means that lots of people believe collectively, what nobody believes individually. (Abba Eban)

\section{Thomas F. Lüschera, Gilbert Abetel ${ }^{b}$}

a Prof. Dr méd., directeur de la clinique de cardiologie, HerzKreislaufZentrum (Centre cardio-circulatoire), Hôpital universitaire de Zurich. L'auteur n'est actuellement pas membre d'un Guidelines Committees, mais conseiller et détenteur de contrats de recherche avec la plupart des entreprises pharmaceutiques et fabricant d'appareils médicaux. Il est en outre Editor-in-chief du European Heart Journal, qui publie toutes les guidelines de l'European Society of Cardiology et édite le ESC Textbooks of Cardiovascular Medicine (Oxford University Press 2009), qui base ses recommandations sur ces guidelines.

b Dr méd., spécialiste en médecine interne, Orbe.
Depuis que l'homme existe, il a besoin des conseils de ses semblables - au début c'était les conseils des aînés, ceux qui avaient survécu dans la nature et semblaient particulièrement dignes de confiance [1]. Avec la naissance du langage, la parole et plus tard l'écriture se sont avérés être un avantage significatif dans la bataille pour la survie - chacun pouvait dès lors profiter des expériences des autres [2]. Aujourd'hui encore, nos parents nous accompagnent à travers notre jeunesse, et par la suite nous sommes guidés par nos amis, nos professeurs et nos modèles professionnels. En bref: les conseils des autres nous sont précieux.

\section{Des impressions aux chiffres}

La médecine, elle aussi, vit de conseils: à l'origine, les grands médecins relataient leurs expériences dans leur pratique clinique et dans leurs ouvrages. Contrairement à aujourd'hui, on transmettait alors ce que l'on avait appris par soi-même et ce qui nous avait été enseigné par nos professeurs [3]; c'était la notoriété de l'auteur qui était convaincante - non pas les chiffres et les statistiques. Encore en 1837, la question de savoir si la médecine devait être considérée comme un art ou comme une science exacte opposait Risueno d'Amador et Pierre Charles Alexandre Louis à l'Académie Royale de Médecine fondée par Louis XVIII en 1820 à Paris [4]. Les uns considéraient chaque patient comme unique et donc chaque traitement comme un acte créatif du médecin impliqué et rejetaient par conséquent les données quantitatives sur les patients et les maladies. «L'homme moyen n'existe pas!», tel était leur crédo [5]. Pierre Charles Alexandre Louis, par contre, affirmait que nous ne pourrions apprendre qu'en chiffrant nos expériences, en déterminant leur valeur et leur importance et en décrivant leurs évolutions lors d'épidémies et de pandémies, ce qui a mené finalement à la médecine scientifique telle que nous la connaissons aujourd'hui [6].

Les chiffres se sont imposés: ce sont aujourd'hui les résultats des grands essais qui dominent la médecine. Ce n'est plus le médecin isolé qui détermine ou essaie de diriger nos actions et nos décisions, mais bien plus des Guidelines Committees, Advisory Boards, Working Groups et des experts en tout genre. Une évolution qui peut, en principe, être saluée; nous savons que notre jugement - dans la mesure où il est basé sur l'expérience quotidienne - repère souvent trop rapidement des causes là où il n'y en a pas, attribue des processus spontanés à nos propres actions et exagère l'importance de cas isolés. Le biais - nos perceptions erronées, souvent inévitables au quotidien - voile la pensée clinique, voire même la rend superflue. C'est justement pour cette raison que les concepts pathophysiologiques et les résultats des essais aveugles et randomisés sont nécessaires comme base de la pratique médicale. En 1948, Austin Bradford Hill, un épidémiologiste anglais, a été le premier à utiliser un essai randomisé qui lui a permis de prouver la meilleure efficacité de la streptomycine par rapport aux traitements usuels de la tuberculose [7]. La seule découverte des mycobactéries comme origine de la tuberculose par Robert Koch et de la streptomycine par Selman Waksman et Albert Schatz n'a pas été suffisante. Ce n'est que la vérification rigoureuse des nouveaux procédés thérapeutiques dans des essais randomisés qui a pu conduire à la médecine basée sur l'évidence, telle que nous la connaissons aujourd'hui [8].

\section{Des conseils aux guidelines}

Depuis, les conseils en médecine se basent, dans la mesure du possible, sur le savoir et non plus sur des expériences personnelles - dans notre monde globalisé, on les appelle guidelines. Les guidelines sont des directives qui indiquent une direction - une direction et non pas un chemin. Elles essaient de nous guider dans notre pratique quotidienne, elles ne sont pas des ordres émanant d'une instance supérieure; les conseils des experts ne sont pas des lois qui doivent être suivis à la lettre, le bon sens du médecin reste essentiel. Toutefois, les guidelines ont une exigence qui dépasse le conseil occasionnel: elles exigent de dispenser l'art médical en fonction de l'état actuel des connaissances.

Mais comment faire? Comment du savoir peut-il être traduit en instructions? Une telle démarche n'est pas aisée: depuis l'époque de la Grèce antique [9] et notamment depuis Kant [10], nous savons que les jugements de fait et les maximes sont deux choses bien différentes. En effet: Partant de l'affirmation «Les statines diminuent le taux de décès, d'infarctus du myocarde et d'accidents vasculaires cérébraux chez les patients atteints d'hypercholestérolémie», on ne peut directement conclure que «Tous les patients ayant un taux de cholestérol élevé devraient être traités par une statine». Et pourquoi pas? Parce que la première affirmation traduit un état de fait et la deuxième est une instruction qui doit déboucher sur une action. Il va de soi que nous cherchons à baser les recommandations sur un avis scientifique, mais la recommandation ellemême exige que la mesure soit considérée comme suffisamment valable dans un système de valeur culturel 
défini, pour être mise en pratique [11]. Nous devons donc être convaincus que l'investissement est à la hauteur de l'utilité et les coûts économiques et médicaux à la hauteur de l'effet escompté. C'est pourquoi les recommandations ne peuvent pas être les mêmes dans tous les pays et dans toutes les cultures; les recommandations dépendent du pays, du système de santé et de l'environnement politique dans lesquels on pratique. Depuis que nous ne pouvons plus nous appuyer sur des valeurs transcendantales, c'est le discours rationnel de citoyens (ou justement de médecins) égaux en droit, appuyé sur des arguments raisonnables, qui détermine les valeurs en vigueur - tel que l'avait exigé Jürgen Habermas pour toutes les questions concernant la société [12]. Certes, la situation était différente autrefois («God send his son and not a committee») [13], mais aujourd'hui les groupes de travail sont devenus indispensables - l'expertocratie domine aussi la médecine, depuis qu'elle est devenue trop florissante pour être maîtrisée individuellement. tension artérielle, le diabète, les hyperlipidémies ou l'ostéoporose. Des guidelines concernant d'autres maladies ne sont que rarement appliquées en médecine de premier recours, mais jouent un rôle important en milieu hospitalier. Pourtant, il existe d'autres limitations: les guidelines se focalisent sur les maladies et n'abordent que rarement le fait que de nombreux patients présentent des diagnostics multiples [16]. Et c'est bien pour cette raison que les guidelines doivent être mises en œuvre par un médecin compétent; il ne s'agit justement pas d'un livre de cuisine pour débutants, mais de directives que le médecin doit adapter au patient individuel avec prudence et sur la base de son expérience.

On est finalement en droit de se demander si des guidelines validés par des boards étrangers sont applicables à nos patients helvétiques. Peut-on soigner la population suisse sur la base de statistiques américaines? A titre d'exemple, on a constaté que le risque absolu lié à une pression sanguine ou à un taux de

\section{«Les guidelines sont des recommandations basées sur l'état actuel \\ des connaissances. Ils sont élaborés à partir de résultats d'études qui ne reflètent que partiellement le quotidien clinique - mais qui restent néanmoins des conseils importants en milieu hospitalier et en cabinet privé»}

\section{De I'utilité des guidelines}

Des guidelines sont-ils vraiment utiles? Pourquoi ne pouvons-nous pas décider nous-mêmes? Finalement, nous avons tous fait des études et accumulé des expériences. Et certes, l'Aufklärung («les Lumières») nous a appris le Sapere aude - «Ose savoir». Kant l'a défini encore plus clairement: «L'Aufklärung, c'est la sortie de l'homme hors de l'état de minorité dont il est luimême responsable» [14]. Ne sommes-nous pas assez adultes pour décider nous-mêmes? L'Aufklärung a-telle échoué? Incontestablement, les écoliers, les étudiants, mais aussi les assistants en formation postgraduée ont besoin d'instruction. Cette assistance, le médecin spécialiste en a-t-il besoin lui aussi ou bien les guidelines ne sont-ils que des diktats inutiles élaborés par des experts? Les progrès de la médecine ont enrichi la science à tel point qu'il est impossible au médecin isolé de garder un aperçu global qui lui permette de prendre la bonne décision dans tous les cas. Les guidelines partent aussi du point de vue que des directives pratiques pour le diagnostic et le traitement de maladies ne peuvent découler que des connaissances scientifiques existantes et non pas d'expériences autistiques [15] - d'où la légitimité des experts.

Les guidelines peuvent être des indicateurs utiles pour les médecins dans leur pratique quotidienne, c'est le cas pour des guidelines concernant l'hyper- cholestérol ou de glucose élevé est réellement plus faible pour les patients vivant en Suisse que pour ceux qui vivent à Framingham ou en Europe du Nord. Toutefois, la relation linéaire entre la pression sanguine, le taux de cholestérol et de glucose reste la même même si c'est à un niveau légèrement inférieur. L'European Society of Cardiology a donc corrigé ses évaluations des risques [17] pour les pays nordiques ainsi que pour l'Europe Centrale et l'Europe du Sud. Toutefois, on ne dispose pas encore de données actuelles pour la Suisse. Il serait alors souhaitable de disposer de plus de données suisses concernant les patients, leur traitement et leur histoire dans le secteur de la médecine de premier recours; ce n'est qu'ainsi que les guidelines pourront être adaptés à la pratique quotidienne, car le risque absolu influence aussi le rapport entre les coûts et l'utilité. La recherche dans les domaines de l'épidémiologie et de la médecine de premier recours est donc nécessaire et elle devrait notamment bénéficier de soutiens financiers plus importants.

\section{Le poids de l'évidence}

Nos connaissances n'ont pas le même niveau dans tous les domaines - nous en savons plus sur le traitement de l'hypertension que sur la prise en charge de patients atteints de sclérose latérale amyotrophique. A la base déjà, le savoir naît dans les conditions les plus diverses: d'essais expérimentaux, de modèles animaux, 
Figure 1

Niveau de la recherche clinique depuis l'information génétique, en passant par l'étude des cellules et des organes, jusqu'au patients et populations

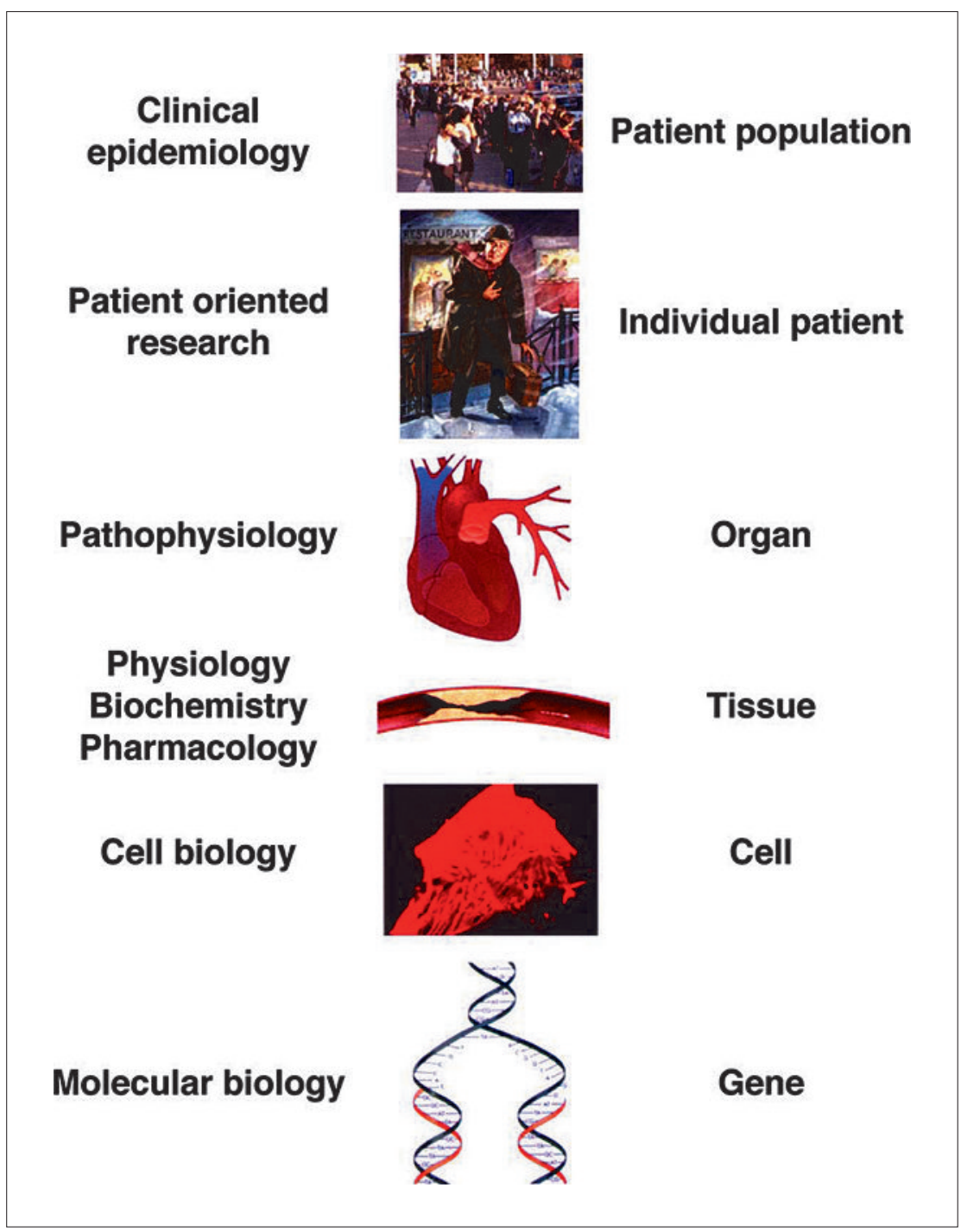

Figure 2

Degré de l'évidence

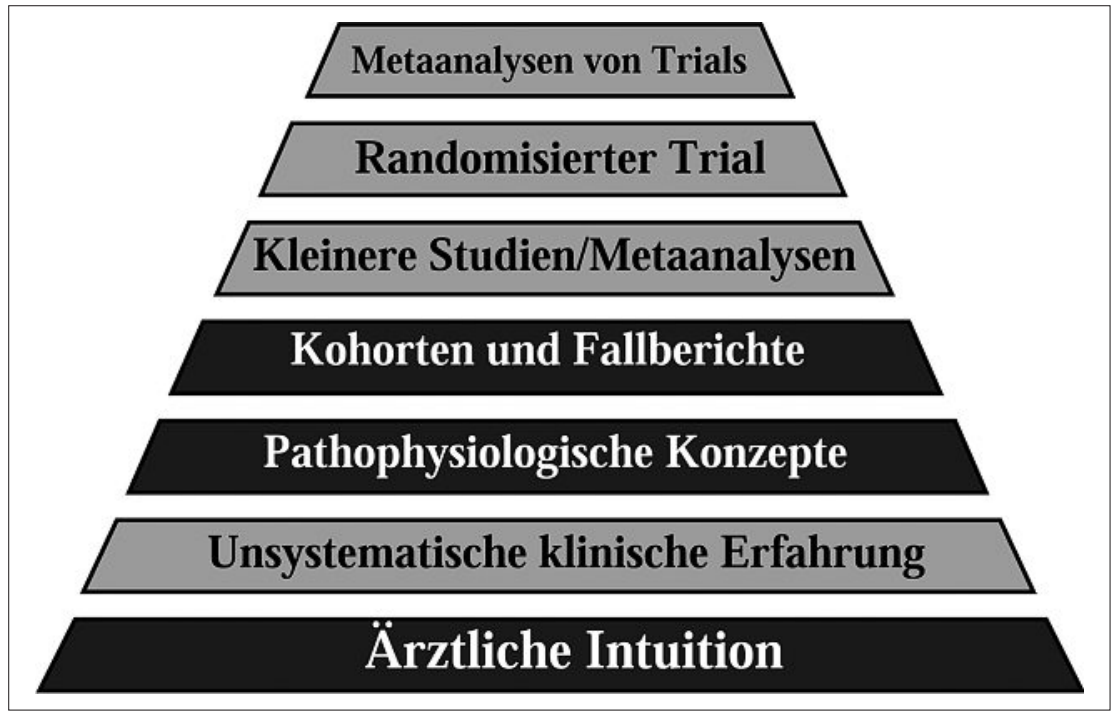

de compte rendus de cas, de petites études proof-ofconcept et finalement de grands groupes de patients (fig. 1). Tous les savoirs n'ont pas la même signification; c'est pourquoi différents niveaux d'évidence ont été définis (fig. 2). Les essais les plus sûrs sont ceux réalisés à partir de plusieurs études randomisées; quelquefois, nous devons nous contenter de petites études, mais pour beaucoup de maladies on ne dispose encore que de sa propre expérience et de quelques cas publiés - les recommandations devront être pondérées en fonction de ces différences (tableau 1, p. 1694).

Mais ce n'est pas encore suffisant: chaque médecin doit encore se demander si le cas de son patient correspond à celui des patients examinés et dans quelle mesure. Pour cela, il est nécessaire de connaître les critères d'inclusion et d'exclusion des grandes études et les données démographiques concernant les patients impliqués; la compétence du médecin traitant reste donc importante, même à l'époque de la médecine basée sur l'évidence, puisque la prise de décision est devenue encore plus difficile.

Disposons-nous de suffisamment d'évidence pour une prise en charge optimale de nos patients? Certainement pas, car dans de nombreux domaines, l'évidence laisse à désirer, voire même est tout à fait insuffisante; nous nous appuyons plutôt sur notre bon sens que sur des données absolues qu'il s'agisse de maladies rares, comme la maladie de Fabry ou la cardiomyopathie hypertrophique, ou bien de pathologies qui relèvent des domaines de la neurologie ou de la médecine générale. Même en médecine cardiovasculaire, nous suivons les recommandations de l'American Heart Association et de l'American College of Cardiology, dont la moitié des directives est encore basée sur un niveau d'évidence C (tableau 1) [18]. Il est donc indispensable de poursuivre les recherches afin d'améliorer la prise en charge des patients. Toutefois, ceci n'est possible qu'avec une collaboration constructive avec l'industrie dans le cadre des dispositions et lois en vigueur, faute de quoi les moyens nécessaires feront défaut. Une condamnation de cette collaboration n'a pas de sens et compromettrait le progrès médical.

\section{Qui est expert?}

Les guidelines committees sont des groupes de travail composés d'experts, de médecins et de chercheurs, c'est-à-dire de personnes disposant d'un savoir spécifique dans un domaine donné. Mais qui est donc expert? La plupart du temps, les guidelines sont édictés par des sociétés comme l'European Society of Cardiology ou la World Gastroenterology Organisation, pour n'en citer que deux, ou des instituts comme NICE (National Institute for Clinical Excellence) ou d'autres encore; ceux-ci déterminent qui sera membre du groupe de travail, qui rédige les guidelines et finalement qui va les publier.

Pour savoir quels experts sont vraiment des experts, on peut aujourd'hui consulter PubMed ou www.isi.org. Toutefois, il est difficile de déterminer si leurs évalua- 
tions sont aussi libres et indépendantes que nous le souhaitons. De nombreux experts sont sans aucun doute impliqués dans des conflits d'intérêt (ou conflicts of interest en nouveau latin), car ils sont très demandés. Non seulement leurs collègues et les institutions publiques veulent profiter de leur savoir, mais également l'industrie compte sur leurs conseils. Il n'existe guère de clinicien ou de chercheur reconnu qui ne soit membre d'un Advisory Board et ne perçoive des honoraires pour des conférences ou la participation à des projets de recherche. Ainsi, non seulement leur compétence de médecin est reconnue, mais les contrats de recherche servent également à leurs propres activités. Selon une étude réalisée en psychiatrie, parmi les 170 membres du panel DSM (Diagnostic and Statistical Manual of Mental Disorders), 95 d'entre eux ont indiqué avoir un lien financier avec l'industrie pharmaceutique [19]. Il convient en outre de relever que les conflits qui influencent notre façon de penser ne sont pas seulement d'ordre financier, mais aussi d'ordre intellectuel [20].

Ces circonstances posent-elles problème? Certes, il est tout à fait envisageable que certains membres de Guidelines Committees - ou même les sociétés responsables elles-mêmes - soient influencés par les soutiens financiers reçus dans d'autres domaines d'activité des fabricants des produits conseillés; il est également concevable que ces mêmes personnes craignent que ces soutiens ne soient remis en question à cause de recommandations négatives. C'est-à-dire que les conflits d'intérêt des membres des commissions sont un réel problème. Comment pouvons-nous remédier à cela? La transparence semble être aujourd'hui la solution appropriée et la mention de tels conflits dans les acknowledgments de ces documents devient donc une nécessité. Il semble toutefois que nous n'ayons pas encore progressé; non seulement les conflits existants

\section{Tableau 1}

Niveau de l'évidence et degré des recommandations dans la médecine basée sur l'évidence

\begin{tabular}{|c|c|c|}
\hline \multicolumn{2}{|c|}{ Niveau de l'évidence } & \multirow{2}{*}{$\begin{array}{l}\text { Recommandation } \\
\text { A }\end{array}$} \\
\hline $1 \mathrm{a}$ & $\begin{array}{l}\text { Métaanalyse resp. révision } \\
\text { systématique des RCT }\end{array}$ & \\
\hline $1 \mathrm{~b}$ & RCT individuel & A \\
\hline $2 a$ & $\begin{array}{l}\text { Révision systématique } \\
\text { d'études de cohortes }\end{array}$ & B \\
\hline $2 b$ & $\begin{array}{l}\text { Etudes individuelles de cohortes } \\
\text { ou RCT de mauvaise qualité }\end{array}$ & B \\
\hline $2 c$ & Etude de recherche des résultats & C \\
\hline 3a & $\begin{array}{l}\text { Révision systématique d'études } \\
\text { de case-control }\end{array}$ & C \\
\hline $3 b$ & $\begin{array}{l}\text { Etudes individuelles } \\
\text { de case-control }\end{array}$ & C \\
\hline 4 & $\begin{array}{l}\text { Séries de cas, études de } \\
\text { case-control, étude de cohorte } \\
\text { de mauvaise qualité }\end{array}$ & C \\
\hline 5 & Avis des experts & D \\
\hline
\end{tabular}

ne sont pas tous mentionnés [21], mais en plus, il est impossible de garantir de cette façon une approche indépendante. Par ailleurs, le type de conflits est lui aussi significatif; les experts qui interviennent dans un nombre important d'entreprises sont plus indépendants que ceux qui ne travaillent qu'avec un seul entrepreneur. Les experts liés à une seule entreprise ne sont pas aptes à être membres de tels Guidelines Committees. En fait, il serait souhaitable d'avoir des experts sans aucun lien, toutefois, en règle générale, ceux-ci ne sont pas les meilleurs dans leur spécialité. Finalement ce sont les recommandations elles-mêmes qui doivent convaincre: La médecine basée sur l'évidence s'appuie sur la logique et sur des données claires; ce qui est vérifiable est convaincant. A cet égard, il s'agit d'être critique dans l'analyse des documents.

\section{Evaluation des recommandations}

Les recommandations doivent donc passer un test de crédibilité: ce qui est important, ce sont les études prises en compte (l'évidence est-elle complète?), leur évaluation (sont-elles randomisées ou en double aveugle? ont-elles été reproduites de façon indépendante?) et la recommandation qui en découle (est-elle adaptée ou exagérée?). L'intégralité de l'évidence peut être entravée par des biais de publication: les études aux résultats négatifs sont moins publiées que celles dont les résultats sont positifs - ceci peut être expliqué par notre attirance pour tout ce qui est intéressant et le penchant des éditeurs à attirer l'attention. Ainsi, un tiers des essais réalisés avec des antidépressifs et enregistrés par la Federal Drug Administration n'a pas été publié [22]. De même, la gestion des données concernant la sécurité cardiovasculaire de Rofecoxib, par Merck, est un exemple de biais de publication [23]. Depuis l'introduction d'un registre des essais cliniques par le gouvernement américain (www. clinicaltrials.gov) et la publication des plans d'études et de manuels pour les essais cliniques [24], la dissimulation de résultats est devenue plus difficile. L'initiative de l'éditeur de revues scientifiques importantes, selon laquelle les Primary Investigators doivent avoir un accès à toutes les données d'un essai clinique, y a également contribué. Grâce au processus de review actuel adopté par les revues scientifiques les plus renommées, avec la collaboration d'experts expérimentés et d'un expert statistique, la qualité des données scientifiques est aujourd'hui bien plus élevée qu'elle ne l'a jamais été. Dès lors, les conflits de membres isolés de ces groupes de travail peuvent être minimisés - probablement pas supprimés - en discutant du problème dès le début du travail, en assurant la transparence et en impliquant un nombre adéquat d'experts. Egalement la comparaison avec des publications d'autres Guidelines Committees renseigne sur le noyau des déclarations: tous les guidelines sontils consistants ou existe-t-il de grandes différences? Finalement, chaque médecin doit décider lui-même 
dans quelle mesure les recommandations peuvent être mises en œuvre ou non dans son environnement et dans le système de la santé dans lequel il exerce.

\section{Que reste-t-il à considérer?}

Les guidelines sont des recommandations basées sur l'état actuel des connaissances. Ils sont élaborés à partir de résultats d'études qui ne reflètent que partiellement le quotidien clinique - mais qui restent néanmoins des conseils importants en milieu hospitalier et en cabinet privé. Il reste à s'interroger dans quelle mesure ils tiennent compte de l'évidence disponible et dans quelle mesure leurs recommandations peuvent prendre appui sur cette évidence. Les conflits de ses auteurs doivent être élucidés et un nombre adéquat d'experts dans chaque groupe de travail doit garantir que ces derniers auront aussi peu d'influence que possible sur ces recommandations. Mais finalement, c'est la vérification de l'évidence à la base et la consistance des recommandations qui en découlent qui doivent être remises en question - la transparence à elle seule ne nous aide pas à avancer, une réflexion indépendante est indispensable, même à l'époque des guidelines.

\section{Références}

1 Canetti E. Masse und Macht. München:Carl Hanser; 1960. S. $267 \mathrm{ff}$.

2 Lüscher TF. Gedankenmedizin. Heidelberg: Springer; 2009. S. 101-110.

3 Heberden W. Some account of a disorder of the breast. Medical Transactions of the Royal College of Physicians of London. 2, 59-67, 1772.

4 Lüscher TF. Gedankenmedizin. Heidelberg: Springer; 2009. S. 77-86.

5 Mathews JT. Quantification and the quest for medical certainty. Ewing, NJ, USA: Princenton University Press; 1995. S14-38.

6 Haynes RB, Sackett D, Guyatt GH, Tugwell P. Clinical epidemiology: How to do clinicl practice research. 3rd edition. Philadelphia: Williams and Wilkins; 2005.

7 Hill AB. Streptomycin treatment of pulmonary tuberculosis: A Medical Research Council investigation. Brit. Med. J. 1948:769-773.
8 Yusuf S, John,.. Cairns A. Camm J, Fallen EL, Gersh BJ: Evidence-based Cardiology, London: BMJ Books; 2003.

9 Aristoteles. Nikomachische Ethik. Zürich: Artemis \& Winkler; 2001.

10 Kant I: Die drei Kritiken. Stuttgart: Alfred Kröner Verlag, S. 1069.

11 Lüscher TF. Gedankenmedizin. Heidelberg: Springer; 2009. S. $81 \mathrm{ff}$.

12 Habermas J. Theorie des kommunikativen Handelns. Frankfurt: Suhrkamp; 1987.

13 Vanhoutte PM. Persönliche Mitteilung 1987.

14 Kant I. Was ist Aufklärung? Hamburg: Meiner Philosophische Bibliothek; 1999, S. 20.

15 Bleuler E. Das autistisch-undisziplinierte Denken in der Medizin und seine Überwindung. Saarbrücken: Verlag Classic Edition; 2009.

16 Shaneyfelt TM, Centor RM. Reassessment of clinicasl practice guidelines - Go gently into that good night. J. Amer. Med. Ass. 2009;301: 868-869.

17 ESC Pocket Guidelines on Cardiovascular Prevention. European Society of Cardiology, Sophia-antipolis, 2007.

18 Tricoci P, Allen JM, Kramer JM, Califf RM, Smith SC. Scientific evidence underlying the ACC/AHA clinical practice guidelines. J. Amer. Coll. Cardiol. 2009;301:831-841.

19 Cosgrove L, Krimsky S, Vijayaraghavan M, Schneider L. Financial ties between DSM-IV panel members and the pharmaceutical industry. 2006;75:154-160.

20 Lüscher TF: Conflicts of Interest oder Interesse am Konflikt? Schweiz Ärztezeitung. 2001;82:2137-2144

21 Okoke K, Kocher MS, Wie EX, Mehlman CT, Bhandari W: Accuracy of conflict-of-interest disclosures reported by physicians. New Engl J Med. 2009;361:1466-1474.

22 Turner EH, Matthews AM, Linardatos E, Tell RA, Rosenthal R. Selective publication of antidepressant trials and its influence on apparent efficacy. NEJM 2008;358:252-260.

23 Topol E: Failing the public health - Rofecoxib, Merck and the FDA. NEJM 2004;351:17-19.

24 Kloner RA, Birnbaum Y. Cardiovascular trials review, 10th edition, Darien, CT, USA: Le Lacq Communication; 2006. 